\title{
Vereinigung der Kassenpsychologen
}

Kontaktadresse: Vereinigung der Kassenpsychologen, Bundesgeschäftsstelle, Cranger Straße 129, D-4650 Gelsenkirchen, Tel. (0209) 76330, Fax (0209) 780499

- Diplom-Psychologen, die berechtigt sind, Psychotherapie zu Lasten der Krankenkassen auszuführen - e.V., Sitz Bonn, gegr. 1984

Bundesv or stand:

Dipl.-Psych. Günter Mehring Gf. B Vors. Dipl.-Psych. Manfred Burkart Dipl.-Psych. HansJochen Weidhaas

Die Vereinigung der Kassenpsychologen e.V. hat im Januar 1991 eine Fragebogenaktion durchge-führt, um einige Übersichtsdaten über psychotherapeutische Leistungen, Therapiebeginn und Wartezeit sowie die Zusammenarbeit mit Ärzten zu ermitteln. Dieser Fragebogen wurde an 440 Mitglieder der Vereinigung der Kassenpsychologen versandt. 67\% (N = 277) der Mitglieder haben den Fragebogen zurückgesandt. Im folgenden werden kurz die Ergebnisse dieser Befragung zusammen mit der Instruktion mitgeteilt.

1. Welche psychotherapeutischen Leistungen erbringen Sie z. Zt.?

Verhaltenstherapie

Tiefenpsychologische Therapie

Psychoanalyse

Erwachsenenpsychotherapie

Psychotherapie mit Kindern und Jugendlichen

Gruppentherapie

270(97\%) 26 (9\%) 12 (4\%)

208(75\%)

(38\%)

$(38 \%)$

2. Wieviele Therapiestunden à 50 min führen Sie z. Zt. wöchentlich im Schnitt durch (einschl. Gruppenstunden)?

0-10 Std./Woche 11-20 Std./Woche 21-30 Std./Woche 31-40 Std./Woche 41-50 Std./Woche 5160 Std./Woche

21 (9\%) 61 (23\%) 95 (36\%) 65 (25\%) 19 (7\%) 2 (1\%)

3. a) Dauer bis zum Beginn einer Einzeltherapie - «Wartezeit» bei Aufnahmestopp ggf. einschätzen?

Mitteilungen der Verbände

bis 3 Monate

bis 6 Monate

9 Monate und länger

Aufnahmestopp

keine Angaben

3. b) Dauer bis zum Beginn einer Probesitzung?

1-3 Wochen 


\section{4-6 Wochen}

7-9 Wochen 10-12 Wochen 13-15 Wochen

$129(46 \%)$

$103(36 \%)$

$36(12 \%)$

$19(5 \%)$

$1(<1 \%)$

123 (49\%) 58(23\%)

$9(4 \%) 37$ (15\%)

$6(1 \%)$

4. Führen Sie in der Regel ein Erstgespräch zur eigenen Indikationsstellung und Feststellung der Dringlichkeit durch?

nein

keine Angaben

$215(77 \%) 60(21 \%)$

$3(<1 \%)$

173

5. Werden Sie zur Krisenintervention sofort therapeutisch tätig?

nein

keine Angaben

$211(76 \%)$

$59(21 \%)$

$6(3 \%)$

6. Bute schätzen Sie die durchschnittliche Bearbeitungsdauer der Langzeitgutachten ein

(Abschicken der Antragstellung bis zum Vorliegen eines Behandlungsausweises).

Monat

Monate

Monate und länger keine Angaben

13 (5\%)

$162(58 \%)$

$95(34 \%)$

$6(3 \%)$

7. Abgesehen von der Tatsache der Delegation ist die Zusammenarbeit mit ArztlÄrztin in der Regel...

unerträglich

schwierig

zufriedenstellend

gut

keine Angaben

$3(1 \%)$

$17(6 \%)$

96(35\%)

$159(57 \%)$

$1(<1 \%)$

8. Was behindert Ihre therapeutische Arbeit in der freien Praxis unter den gegebenen Verhältnissen am meisten? 
Am häufigsten genannt wurden:

Delegation Gutachten schreiben bürokratischer Aufwand

9. Sind Sie bereit, eíne unterschiedliche Honorierung der gleichen Leistung, von Arzt oder psycholo-gischen Psychotherapeuten erbracht, zu akzeptieren?

ja

nein

keine Angaben

(1\%) 270(98\%)

$(1 \%)$

Verhaltenstherapie 1991; 1:173-174

Verein zur Förderung der klinischen Verhaltenstherapie (VFKV)

Nach der Anerkennung des Instituts und den Vorgaben der Kassenärztlichen Bundesvereinigung wurden die Voraussetzungen für die berufsbegleitende Ausbildung der Teilnehmer erweitert und eine Institutsambulanz unter der Bezeichnung «Verhaltensmedizinische Ambulanz» geschaffen. Die Ambulanz nimmt beratende und therapeutische Aufgaben wahr, wobei die Finanzierung durch freiwillige Beiträge der Klienten/Patienten erfolgen soil. Die Erstgespräche werden jeweils mit einem anerkannten Supervisor und einem Ausbildungskandidaten am Institut durchgeführt. Die weitere Supervision soil durch den Supervisor, der auch am Erstgespräch teilnahm, erfolgen. Bei den Supervisoren des Instituts handelt es sich um Allgemeinärzte, Fachärzte verschiedener Disziplinen und klinische Psychologen, so daß eine umfassende Berücksichtigung aller relevanten verhaltensmedizinischen Aspekte möglich sein wird, nachdem in der Regel das Hauptproblem des Patienten schon bei der Anmeldung erfaßt wird. Geplant ist eine Dokumentation über soziodemoMitteilungen der Verbände 